PROCEEDINGS OF THE

AMERICAN MATHEMATICAL SOCIETY

Volume 134, Number 7, Pages 1869-1873

S 0002-9939(05)08194-3

Article electronically published on December 19, 2005

\title{
FAILURE OF KRULL-SCHMIDT FOR INVERTIBLE LATTICES OVER A DISCRETE VALUATION RING
}

\author{
ESTHER BENEISH
}

(Communicated by Martin Lorenz)

\begin{abstract}
Let $p$ be a prime greater than 3 , and let $N$ be the semi-direct product of a group $H$ of order $p$ by a cyclic $C$ group of order $p-1$, which acts faithfully on $H$. Let $R$ be the localization of $Z$ at $p$. We show that the Krull-Schmidt Theorem fails for the category of invertible $R N$-lattices.
\end{abstract}

\section{INTRODUCTION}

Let $G$ be a finite group and let $R G$ be the group ring of $G$ with coefficients in a Dedekind domain $R$. An $R G$-lattice $M$ is defined to be a finitely generated $R$-torsion-free $R G$-module. $M$ is said to be a permutation lattice if it is $R$-free and has an $R$-basis permuted by $G . M$ is said to be an invertible or a permutation projective lattice, if it is a direct summand of a permutation lattice. This note was motivated by a question of A. Merkurjev about the existence of a category of invertible lattices over a discrete valuation ring, for which the Krull-Schmidt Theorem failed. The question arose in the study of the problem of the uniqueness of a direct sum decomposition of the motive of a projective homogeneous variety into indecomposable objects in the category of Chow motives. This category contains a subcategory equivalent to the category of invertible lattices for a certain finite group. Failure of Krull-Schmidt for this subcategory implies failure of uniqueness of direct sum decompositions for the motives; see [4].

Let $p$ be a prime greater than 3 , and let $N$ be the semi-direct product of a group $H$ of order $p$ by a cyclic group $C$ of order $p-1$, where $C$ acts faithfully on $H$ by conjugation. Let $R$ denote the localization of the ring of integers, $Z$, at the prime $p$. We show that the Krull-Schmidt Theorem fails for the category of invertible $R N$-lattices.

\section{INVERTIBLE LATTICES}

Let $N=H \rtimes C$ be as defined in the introduction. The $Z N$-lattice $Z N / H$ is isomorphic to $Z C$, and $Z H$ has the structure of a $Z N$-lattice via the isomorphism

$$
Z N / C \cong Z H \text {. }
$$

Received by the editors September 8, 2004 and, in revised form, October 24, 2004 and February $5,2005$.

2000 Mathematics Subject Classification. Primary 20C10, 20C11.

Key words and phrases. Permutation lattices, invertible lattices, Krull-Schmidt.

This research was partially supported by NSF grant \#DMS-0244766. 
Let $I_{H}$ be the augmentation ideal of $Z H$. We have the following $Z N$-exact sequences:

$$
0 \rightarrow I_{H} \rightarrow Z H \rightarrow Z \rightarrow 0 .
$$

Tensoring by $I_{H}$ over $Z$ and setting $V=I_{H} \otimes I_{H}$ we obtain

$$
0 \rightarrow V \rightarrow Z H \otimes I_{H} \rightarrow I_{H} \rightarrow 0 .
$$

One checks directly that $\operatorname{Res}_{C}^{N} I_{H} \cong Z C$, and so the following isomorphisms are given by Frobenius reciprocity:

$$
Z H \otimes I_{H} \cong Z N \otimes_{Z C} \operatorname{Res}_{C}^{N} I_{H} \cong Z N \otimes_{Z C} Z C \cong Z N .
$$

Therefore, (2.1) becomes

$$
0 \rightarrow V \rightarrow Z N \rightarrow I_{H} \rightarrow 0 .
$$

For any $Z N$-lattice $M$, we let $M^{*}=\operatorname{Hom}(M, Z)$. Let $q$ be a prime different from $p$, and let $C_{q}$ be a $q$-Sylow subgroup of $N$. Without loss of generality, we may assume that $C_{q}$ is contained in $C$. Thus $H^{1}\left(C_{q}, I_{H}\right) \cong H^{1}\left(C_{q}, Z C\right)=0$. We also have $H^{1}\left(H, I_{H}\right) \cong Z / p Z$, and hence $H^{1}\left(H, I_{H}^{*}\right) \cong Z / p Z$. Let $\alpha$ generate $H^{1}\left(H, I_{H}^{*}\right)$. By [6. Proposition 12.5] there exists a $Z N$-lattice $W$ and an exact sequence

$$
0 \rightarrow I_{H}^{*} \rightarrow W^{*} \rightarrow Z N / H \rightarrow 0
$$

such that the image of $\alpha$ in $H^{1}\left(H, W^{*}\right)$ is 0 , and hence $W^{*}$ is $H^{1}$-trivial since $H^{1}\left(N, I_{H}^{*}\right)$ injects into $H^{1}\left(H, I_{H}^{*}\right)$. Since $N$ is meta-cyclic this implies that $W^{*}$ is invertible by [3, Proposition 2, section 1]. Dualizing the above sequence we obtain

$$
0 \rightarrow Z N / H \rightarrow W \rightarrow I_{H} \rightarrow 0
$$

with $W$ invertible.

Lemma 2.1. There is an isomorphism of $Z N$-lattices

$$
V \oplus W \cong Z N \oplus Z N / H
$$

Proof. We form the following pullback diagram with sequences (2.2) and (2.3):

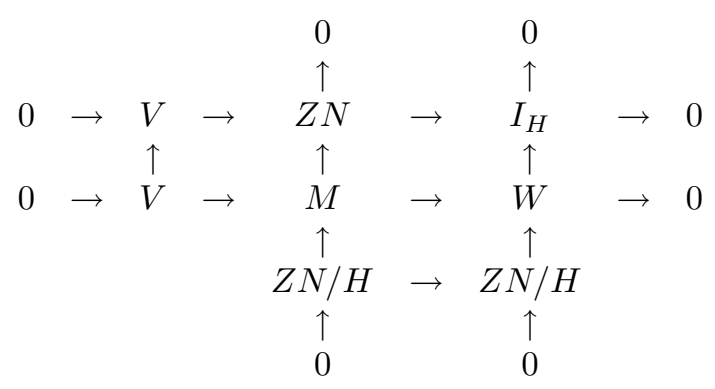

We have $\left(I_{H}\right)^{K}=0$ for all subgroups $K$ of $N$ so that $V$ is $H^{1}$-trivial. Since $N$ is meta-cyclic, this implies that $V$ is invertible by [3, Proposition 2, section 1] By [3. Lemma 9, section 1], the middle horizontal and vertical sequences split, which proves the lemma.

Notation 2.2. For a $Z N$-lattice $M$, let $M_{p}$ denote its localization at the prime $p$, and let $\hat{M}$ denote its $p$-adic completion. 
Remark 2.3. We have

$$
\hat{Z} N / H \cong \hat{Z}[x] /\left(x^{p-1}-1\right) \cong \bigoplus_{k=1}^{p-1} \hat{Z}[x] /\left(x-\theta^{k}\right) \cong \bigoplus_{k=1}^{p-1} Z_{k}
$$

where $Z_{k} \cong \hat{Z}[x] /\left(x-\theta^{k}\right)$ and $\theta$ is a primitive $(p-1)^{s t}$ root of 1 in $\hat{Z}$. So $Z_{k}$ is a $\hat{Z} N$-module of $\hat{Z}$-rank 1 with trivial $H$-action and such that if $c$ generates $C$, then $c .1=\theta^{k}$. Therefore,

$$
\hat{Z} N \cong \hat{Z} H \otimes \hat{Z} C \cong \bigoplus_{k=1}^{p-1} \hat{Z} H \otimes Z_{k} .
$$

Note that for each $k, \hat{Z} H \otimes Z_{k}$ is indecomposable since $\operatorname{Res}_{H}^{N} \hat{Z} H \otimes Z_{k} \cong \hat{Z} H$, and $\hat{Z} H$ is an indecomposable $\hat{Z} H$-module by [2, Corollary 19.24].

Theorem 2.4. Let $R$ denote the localization of $Z$ at the prime $p$. Then the KrullSchmidt Theorem fails for invertible RN-lattices.

Proof. By [1, Theorem 2.3] we have

$$
\hat{V} \cong\left(\bigoplus_{k=2}^{p-1} \hat{Z} H \otimes Z_{k}\right) \oplus Z_{1}
$$

Therefore by Lemma 2.1 and Remark 2.3

$$
\hat{W} \cong\left(\bigoplus_{k=2}^{p-1} Z_{k}\right) \oplus \hat{Z} H \otimes Z_{1} .
$$

Let $Q$ be the field of rational numbers. For each $k$ dividing $p-1$, let $\omega_{k}$ be a primitive $k^{t h}$ root of 1 over $Q$. Then

$$
Q N / H \cong \bigoplus_{k \mid p-1} Q\left(\omega_{k}\right)
$$

and the $Q\left(\omega_{k}\right)$ are the irreducible components of $Q N / H$. Now $Z N / H$ is isomorphic to $Z C$ as a $Z N$-module, and since $R C$ is a maximal $R$-order in $Q C$ we have

$$
R N / H \cong \bigoplus_{k \mid p-1} R\left[\omega_{k}\right]
$$

by [2, Proposition 31.2]. Therefore,

$$
R N \cong \bigoplus_{k \mid p-1} R H \otimes R\left[\omega_{k}\right]
$$

Now for each $k$ we have

$$
\hat{Z}\left[\omega_{k}\right] \cong \hat{Z}[x] / \phi_{k}(x)
$$

where $\phi_{k}(x)$ is the $k^{\text {th }}$ cyclotomic polynomial. As above we let $\theta \in Q_{p}$ be a primitive $(p-1)^{s t}$ root of 1 over $Q$, where $Q_{p}$ is the completion of $Q$ at the prime $p$. Set $\omega_{k}=\theta^{(p-1) / k}$ and let $J_{k}=\{i \in Z: 1 \leq i<k,(i, k)=1\}$. Then $\phi_{k}(x)=\prod_{j \in J_{k}}\left(x-\omega_{k}^{j}\right)$. Therefore,

$$
\hat{Z}\left[\omega_{k}\right]=\bigoplus_{j \in J_{k}} Z_{j} .
$$


Consequently,

$$
\hat{V} \cong\left(\bigoplus_{k \mid p-1, k \neq p-1} \hat{Z} H \otimes \hat{Z}\left[\omega_{k}\right]\right) \oplus\left(\bigoplus_{k \in J_{p-1}, k \neq 1} \hat{Z} H \otimes Z_{k}\right) \oplus Z_{1}
$$

and

$$
\hat{W} \cong\left(\bigoplus_{k \mid p-1, k \neq p-1} \hat{Z}\left[\omega_{k}\right]\right) \oplus\left(\bigoplus_{k \in J_{p-1}, k \neq 1} Z_{k}\right) \oplus \hat{Z} H \otimes Z_{1} .
$$

To simplify notation set

$$
M=\bigoplus_{k \mid p-1, k \neq p-1} R H \otimes R\left[\omega_{k}\right] \text { and } M^{\prime}=\bigoplus_{k \mid p-1, k \neq p-1} R\left[\omega_{k}\right] .
$$

Since $\hat{Z}$ is a faithfully flat $R$-module by [5, Corollary 2.2] we have $\hat{V} / \hat{M} \cong V_{p} \hat{/} M$. Similarly, $\hat{W} / \hat{M}^{\prime} \cong W_{p} \hat{/} M^{\prime}$. Therefore, the $R N$-lattices $S=V_{p} / M$ and $S^{\prime}=$ $W_{p} / M^{\prime}$ have the property that

$$
\hat{S}=\left(\bigoplus_{\left(k \in J_{p-1}, k \neq 1\right.} Z_{k} \otimes \hat{Z} H\right) \oplus Z_{1}
$$

and

$$
\hat{S}^{\prime}=\left(\bigoplus_{k \in J_{p-1}, k \neq 1} Z_{k}\right) \oplus \hat{Z} H \otimes Z_{1} .
$$

We have $\hat{V}=\hat{M} \oplus \hat{S}$ and $\hat{W}=\hat{M}^{\prime} \oplus \hat{S}^{\prime}$, which implies that $V_{p}=M \oplus S$ and $W_{p}=M^{\prime} \oplus S^{\prime}$, 2, Proposition 30.17]. Since $V_{p} \oplus W_{p}=R N / H \oplus R N$ by Lemma 2.1, $S$ and $S^{\prime}$ are invertible $R N$-lattices. Since

$$
\hat{Z}\left[w_{p-1}\right]=\bigoplus_{k \in J_{p-1}} Z_{k},
$$

we get

$$
\hat{S} \oplus \hat{S}^{\prime} \cong \hat{Z} H \otimes \hat{Z}\left[\omega_{p-1}\right] \oplus \hat{Z}\left[\omega_{p-1}\right],
$$

and so by [2, Proposition 30.17],

$$
S \oplus S^{\prime} \cong R H \otimes R\left[\omega_{p-1}\right] \oplus R\left[\omega_{p-1}\right] .
$$

Now $R\left[\omega_{p-1}\right]$ is indecomposable since $Q\left(\omega_{p-1}\right)$ is irreducible, but it is not a direct summand of either $S$ or $S^{\prime}$, for if it were, then $\hat{Z}\left[\omega_{p-1}\right]$ would be a direct summand of $\hat{S}$ or $\hat{S}^{\prime}$ which is a contradiction.

Note that the condition, that the prime $p$ be greater than 3 , is necessary for if $p=3$, then $S=Z\left[\omega_{2}\right]$ and if $p=2$, then $S=R=Z\left[\omega_{1}\right]$.

\section{ACKNOWLEDGMENT}

The author would like to thank the referee for a very thorough study of the manuscript. 


\section{REFERENCES}

[1] E. Beneish, Monomial actions of the symmetric group, J. of Alg. 265 (2003) 405-419. MR1987009 (2004b:20008)

[2] C. Curtis and I. Reiner, Methods of Representation Theory, Vol. 1, Springer-Verlag, 1982. MR0632548 (82i:20001)

[3] J.-L. Colliot-Thelene and J.-P. Sansuc, La R-equivalence sur les tores, Ann. Sci. Ecole Normale Sup. (4) 10 (1977), 175-230. MR0450280 (56:8576)

[4] V. Chernousov and A. Merkurjev, Motivic decomposition of projective homogeneous varities and the Krull-Schmidt Theorem, preprint.

[5] I. Reiner, Maximal Orders, Academic Press, 1975.

[6] D. Saltman, Lectures on Division Algebras, CMBS, Regional Conference series in Mathematics, number 94, 1998. MR.1692654 (2000f:16023)

Department of Mathematics, Central Michigan University, Mount Pleasant, MichiGAN 48859

E-mail address: benei1e@cmich.edu 